bowel enterocytes. J. Clin. Invest. 90:1871-1878.

5. Kolars, J.C., et al. 1991. First-pass metabolism of cyclosporin by the gut. Lancet 338:1488-1490.

6. Paine, M.F., et al. 1996. First-pass metabolism of midazolam by the human intestine. Clin. Pharmacol. Ther. 60:14-24.

7. Guengerich, F.P. 1999. Cytochrome P-450 3A4: regulation and role in drug metabolism. Annu. Rev. Pharmacol. Toxicol. 39:1-17.

8. van Herwaarden, A.E., et al. 2007. Knockout of cytochrome P450 3A yields new mouse models for understanding xenobiotic metabolism. J. Clin. Invest. 117:3583-3592. doi:10.1172/JCI33435.

9. Cong, D., Doherty, M., and Pang, K.S. 2000. A new physiologically based, segregated-flow model to explain route-dependent intestinal metabolism. Drug Metab. Dispos. 28:224-235.

10. Paine, M.F., et al. 1997. Characterization of interand intra-intestinal differences in human CYP3Adependent metabolism. J. Pharmacol. Exp. Ther.
283:1552-1562.

11. von Richter, O., et al. 2004. Cytochrome P450 3A4 and P-glycoprotein expression in human small intestinal enterocytes and hepatocytes: a comparative analysis in paired tissue specimens. Clin. Pharmacol. Ther. 75:172-183.

12. Thummel, K.E., and Wilkinson, G.R. 1998. In vitro and in vivo drug interactions involving human CYP3A. Annu. Rev. Pharmacol. Toxicol. 38:389-430.

13. Amidon, G.L., et al. 1995. A theoretical basis for a biopharmaceutic drug classification: the correlation of in vitro drug product dissolution and in vivo bioavailability. Pharm. Res. 12:413-420.

14. Wu, C.Y., and Benet, L.Z. 2005. Predicting drug disposition via application of BCS: transport/absorption/ elimination interplay and development of a biopharmaceutics drug disposition classification system. Pharm. Res. 22:11-23.

15. Lown, K.S., et al. 1997. Role of intestinal P-glycoprotein $(\mathrm{mdr} 1)$ in interpatient variation in the oral bioavailability of cyclosporine. Clin. Pharmacol. Ther. 62:248-260.

16. Wacher, V.J., et al. 1998. Role of P-glycoprotein and cytochrome P450 3A in limiting oral absorption of peptides and peptidomimetics. J. Pharm. Sci. 87:1322-1330.

17. Cheung, C., et al. 2006. Growth hormone determines sexual dimorphism of hepatic cytochrome P450 3A4 expression in transgenic mice. J. Pharmacol. Exp. Ther. 316:1328-1334.

18. Thummel, K.E., et al. 2001. Transcriptional control of intestinal cytochrome P-4503A by $1 \alpha, 25$-Dihydroxy Vitamin D $\mathrm{D}_{3}$. Mol. Pharmacol. 60:1399-1406.

19. Jurutka, P.W., et al. 2005. Molecular and functional comparison of 1,25-dihydroxyvitamin $\mathrm{D}_{3}$ and the novel vitamin D receptor ligand, lithocholic acid, in activating transcription of cytochrome P450 3A4. J. Cell. Biochem. 94:917-943.

20. Makishima, M., et al. 2002. Vitamin D receptor as an intestinal bile acid sensor. Science. 296:1313-1316.

\title{
Cardiac growth and angiogenesis coordinated by intertissue interactions
}

\author{
Kenneth Walsh ${ }^{1}$ and Ichiro Shiojima ${ }^{2}$
}

\begin{abstract}
${ }^{1}$ Molecular Cardiology Unit, Whitaker Cardiovascular Institute, Boston University School of Medicine, Boston, Massachusetts, USA. ${ }^{2}$ Department of Cardiovascular Science and Medicine, Chiba University School of Medicine, Chiba, Japan.
\end{abstract}

\begin{abstract}
Cardiac hypertrophy and angiogenesis are coordinately regulated during physiological or adaptive cardiac growth, and disruption of the balanced growth and angiogenesis leads to contractile dysfunction and heart failure. Coordination of growth and angiogenesis is in part mediated by the secretion of angiogenic growth factors from myocytes in response to hypertrophic stimuli, which enables the vasculature to "catch up" to the growth of the myocardium. In this issue of the JCI, two studies provide novel insights into the regulatory mechanisms of cardiac growth and coronary angiogenesis. Heineke et al. demonstrate that GATA4 acts as a stress-responsive transcription factor in murine cardiac myocytes that induces the expression of angiogenic growth factors (see the related article beginning on page 3198). Tirziu et al. show that enhanced coronary angiogenesis per se leads to hypertrophic growth of myocytes through a nitric oxide-dependent mechanism (see the related article beginning on page 3188 ). These studies, together with previous reports, suggest the existence of reciprocal signals between the myocardium and the vasculature that promote the growth of each other in a paracrine fashion.
\end{abstract}

Postnatal growth of the heart is primarily achieved through hypertrophy of individual myocytes. Cardiac hypertrophy observed in patients with hemodynamic overload (e.g., hypertension or valvular heart diseases) is referred to as "pathological" because the increased cardiac mass is

Nonstandard abbreviations used: HIF-1, hypoxiainducible factor-1; mTOR, mammalian target of rapamycin.

Conflict of interest: The authors have declared that no conflict of interest exists.

Citation for this article: J. Clin. Invest. 117:3176-3179 (2007). doi:10.1172/JCI34126. associated with increased morbidity and mortality, and sustained overload eventually leads to contractile dysfunction and heart failure (1). However, cardiac hypertrophy is not necessarily pathological. Cardiac growth during normal postnatal development or the hypertrophy observed in trained athletes is referred to as "physiological" because these forms of hypertrophy are not associated with impairment of contractility, and they are morphologically and molecularly distinct from pathological hypertrophy (2). Therefore, two related issues in the field of cardiovascular research concern the mechanisms by which sustained hemodynamic overload leads to heart failure and the mechanisms that distinguish between physiological and pathological cardiac growth.

It was previously reported that physiological cardiac hypertrophy is associated with normal or increased numbers of myocardial capillaries, whereas pathological hypertrophy is correlated with a reduction in capillary density (3). These observational studies suggest that coronary neoangiogenesis occurs during physiological hypertrophy and that vascular rarefaction in the setting of pathological hypertrophy may cause tissue hypoxia and lead to contractile dysfunction. In fact, in a mouse model of Aktinduced myocardial hypertrophy, it was shown that hypertrophic stimuli induced the expression of angiogenic growth factors such as VEGF in the adaptive phase of hypertrophy, and that blockade of VEGF signaling resulted in a reduction in capillary density and an early transition to heart failure (4). In a pressure overload-induced hypertrophy model, VEGF deficiency leads to a reduction in myocardial capillary density and accelerates the transition from compensatory hypertrophy to failure (5). On the other hand, supplementation of VEGF during sustained pressure overload preserves contractile function (6-8). Mech- 


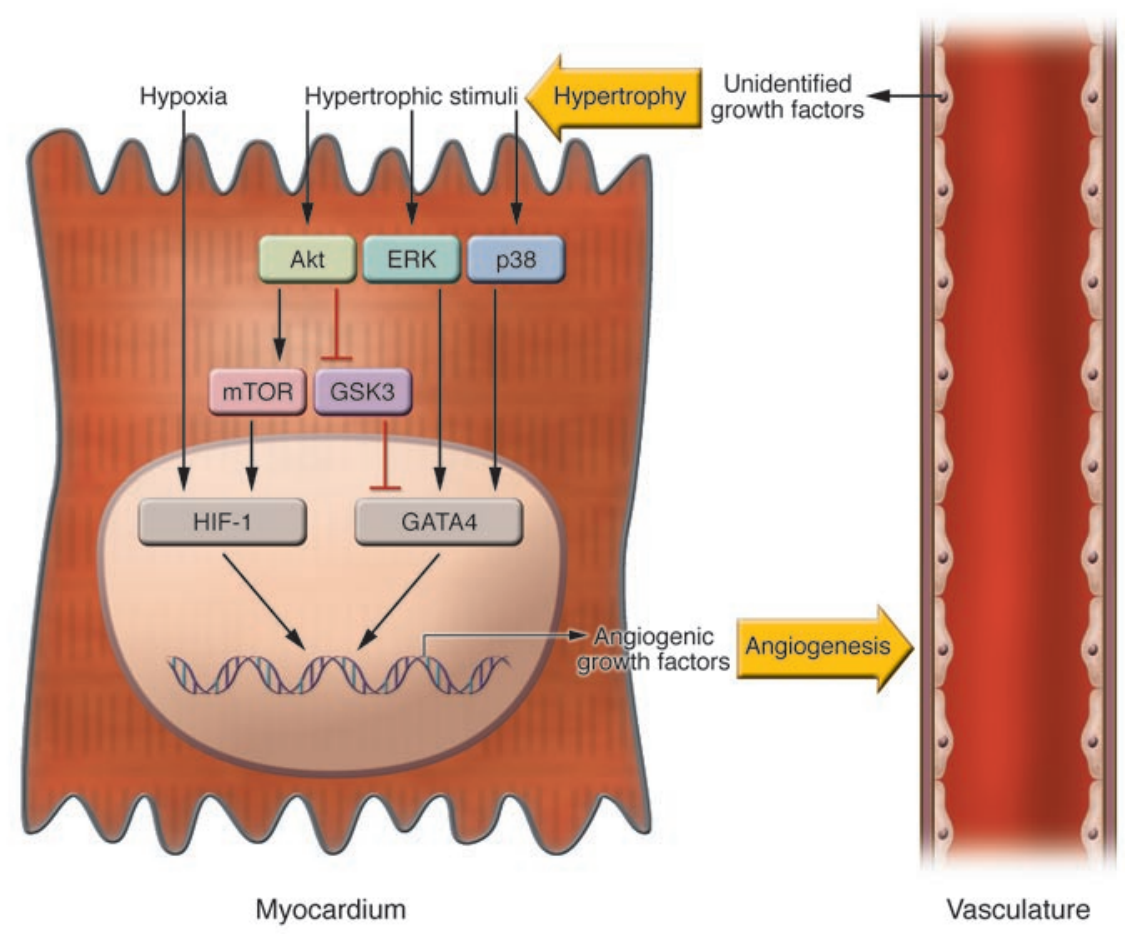

Figure 1

Reciprocal signals between the myocardium and the vasculature coordinately regulate cardiac growth and coronary angiogenesis. In the myocardium (left), hypertrophic stimuli activate multiple protein kinases such as Akt, ERK, and p38, which upregulate the transcriptional activity of GATA4 either directly or indirectly. At the same time, tissue hypoxia induced by cardiac growth, or activation of the Akt-mTOR pathway, stabilizes HIF-1. Activation GATA4 and HIF-1 in the nucleus leads to the expression of angiogenic growth factor genes, which act on the vasculature (right) to induce angiogenesis. The vasculature, on the other hand, when stimulated with angiogenic growth factors, secrets unidentified growth factor(s), which act on the myocardium to promote hypertrophy. Thus coordinated hypertrophy and angiogenesis is mediated by reciprocal signals between the myocardium and the vasculature in a paracrine fashion.

anistically, it was shown that the downregulation of hypoxia-inducible factor-1 (HIF-1) by the p53 tumor suppressor protein, which is induced in the myocardium in the late phase of pressure overload-induced hypertrophy, contributes to the reduced expression of angiogenic growth factors (8). These studies suggest that, in compensatory or physiological cardiac hypertrophy, growth-promoting signals simultaneously induce hypertrophy and angiogenic growth factor expression in myocytes and maintain the balance between myocyte hypertrophy and coronary angiogenesis. These studies also suggest that impaired expression of myocardial angiogenic growth factors in the pathological phase of pressure overload-induced cardiac hypertrophy results in vascular rarefaction and has a causative role in the development of contractile dysfunction. Consistent with this hypothesis, clinical dilated cardiomyopathy is associated with an irregular capillary pattern and a reduction in overall capillary density
$(9,10)$. Thus the balance between growth and angiogenesis in the heart is one of the determinants of whether the hypertrophy is physiological/adaptive or pathological/ maladaptive, and impaired angiogenesis during sustained growth stimuli is critical for the progression from adaptive to maladaptive cardiac hypertrophy (11).

\section{GATA4 is an angiogenic transcription factor in the heart}

In this issue of the JCI, Heineke et al. (12) report that the cardiac transcription factor GATA4 promotes coronary angiogenesis in the murine heart. GATA4 is a zinc-finger transcription factor that is abundantly expressed in cardiomyocytes from early embryonic stages to adulthood and regulates cardiac-specific gene expression (13). Heineke et al. first show that transgenic overexpression of GATA4 in murine adult cardiomyocytes enhances coronary angiogenesis, whereas myocyte-specific deletion of GATA4 results in a reduction in capil- lary density. They further demonstrate that myocytes overexpressing GATA4 can induce in vitro angiogenesis in a paracrine fashion through the secretion of angiogenic growth factors such as VEGF-A, and that GATA4 binds to and transactivates the Vegf-A gene promoter. Finally, Heineke et al. show that ectopic expression of GATA4 in skeletal muscle is sufficient to enhance angiogenesis in a hindlimb ischemia model. Together with the previous observations that the transcriptional activity of GATA4 is enhanced by hypertrophic stimuli (14-16), the results of the study by Heineke et al. suggest that GATA4 plays a role in the induction of hypertrophy-associated coronary angiogenesis.

It should be noted that activation of GATA4 and HIF-1 may represent different mechanisms by which coronary angiogenesis is coordinated with cardiac growth. Activation of GATA4 is predominantly hypoxia independent and is induced by hypertrophic stimuli that are mediated by ERK, p38, or Akt signaling (Figure 1). The phosphorylation of GATA4 by ERK or $\mathrm{p} 38$ potentiates its transcriptional activity $(15,16)$, whereas inhibition of glycogen synthase kinase $3-\beta$ (GSK3- $\beta$ ) by Akt leads to GATA4 activation and results in its accumulation in the nucleus (14). In contrast, HIF-1 is activated by hypoxia that presumably occurs secondary to the extensive growth of myocytes, although it is reported that HIF-1 can also be activated by growth factor signaling through a mammalian target of rapamycin-dependent (mTOR-dependent) pathway that is hypoxia independent (17). Furthermore, GATA4 and HIF-1 activation of the VEGF promoter appears to be mediated by distinct promoter regulatory elements $(8,12)$. Similarly, VEGF activation during growth factor/Akt-mediated skeletal muscle hypertrophy does not involve the HIF-1 cisregulatory element in the VEGF promoter (18). Thus while it appears that multiple mechanisms can coordinate an angiogenic response with myocyte growth, these regulatory programs may crosstalk through proteins that are shared between the different signaling pathways (Figure 1).

Given that angiogenic growth factor expression is downregulated in the chronic phase of hypertrophy, one intriguing question raised by Heineke et al. (12) is how GATA4 is inactivated during sustained pressure overload and whether p53 is involved in this process. Another question is whether there is synergism between GATA4 
and HIF-1 in the transcriptional activation of angiogenic growth factors. Furthermore, capillary density is reduced and tissue hypoxia is detected in the myocardium of myocyte-specific GATA4 knockout mice at baseline, but HIF-1-responsive genes are not activated in this situation. These data indicate that the transcriptional activity of HIF-1 may be impaired in the absence of GATA4 and suggest the possibility of interactions between GATA4 and HIF-1 in the regulation of their common target genes.

\section{Enhanced angiogenesis per se promotes hypertrophy}

In a separate study also in this issue of the JCI, Tirziu et al. provide evidence showing that an increase in capillary mass is sufficient to promote hypertrophic growth of murine myocytes (19). These findings will undoubtedly be controversial because, while it has been fairly well established that a limitation of angiogenic factors will impair cardiac growth and function in response to developmental $(20,21)$ and pathological $(4,5,8)$ stimuli, it does not necessarily follow that an increase in angiogenesis will activate heart growth in the absence of cardiomyocyte growth signals. Many studies have delivered angiogenic growth factors to the heart. For example, the conditional overexpression of VEGF in cardiomyocytes leads to the growth of an abnormal vascular network and edema, but an effect on cardiac hypertrophy was not reported (22). Tirziu et al. first established a transgenic mouse model in which PR39, a naturally occurring antibacterial peptide that inhibits the proteasome-dependent degradation of HIF-1, is expressed in cardiomyocytes in an inducible manner. Using this model, they show that induction of PR39 expression in adult cardiomyocytes for three weeks results in enhanced coronary angiogenesis and increased capillary mass without an increase in heart size or heart weight/body weight ratio. However, when PR39 expression was maintained for six weeks, there was an approximately $40 \%$ increase in heart weight/body weight ratio that was associated with a normalization of vascular density. Because PR39 exhibits no direct prohypertrophic effect on cardiomyocytes in vitro, these results suggest that cardiac growth occurs secondary to an increase in capillary mass (23).

One intriguing question raised by the Tirziu et al. (19) study is how enhanced angiogenesis promotes cardiac hypertrophy. It is possible that newly formed ves- sels supply excessive nutrients and thereby promote hypertrophic growth or that paracrine factors released from the vascular endothelium induce cardiomyocyte hypertrophy. The observation that a nitric oxide synthase inhibitor, L-NAME, partially suppressed angiogenesis-driven cardiac hypertrophy suggests that nitric oxide may be an endothelium-derived prohypertrophic factor. However, this does not necessarily exclude the possibility that other growth factors secreted from endothelial cells also contribute to angiogenesis-induced cardiac growth. Also of interest is the observation that cardiac hypertrophy induced by long-term PR39 expression is pathologi$\mathrm{cal} /$ maladaptive because it is associated with fetal cardiac gene induction, myocyte apoptosis, and decreased contractility. Thus PR39-induced angiogenesis in the absence of hypertrophic stimuli may lead to the "stress" of the myocardium, and it is possible that cardiomyocyte growth in this model may occur in response to injury such as edema or inflammation, in addition to the increase in the capillary bed.

\section{Intertissue interactions that coordinate organ growth and angiogenesis}

The close relationship between tissue growth and angiogenesis appears to be conserved in other organs as well. Tumor growth is angiogenesis dependent (24), and adipose tissue mass can also be regulated by the vasculature (25). Coronary angiogenesis during cardiac growth is enhanced by angiogenic growth factors released from cardiomyocytes $(4,5,8,12)$, and now it is shown that newly formed blood vessels may secrete factors that promote the growth of cardiomyocytes (19). Thus it appears that crosstalk between the myocardium and the vasculature functions to promote the growth of each in a paracrine fashion (Figure 1) (11). This notion has support from earlier studies showing that prostate vessel growth stimulated by testosterone treatment precedes by several days the increase in prostate mass (26), and that hepatocyte growth factor secreted from the liver endothelium in response to VEGF stimulation promotes hepatocyte proliferation (27).

In summary, it is becoming increasingly clear from a number of studies that, in response to hypertrophic stimuli, both heart growth and function are angiogenesis dependent. These findings are important because they challenge the myocytecentric view of cardiac dysfunction and indicate that endothelial cell-myocyte interactions should be taken into account to better understand the pathophysiology of heart failure. Specifically, these studies provide experimental evidence to suggest that a disruption of coordinated myocyte growth and angiogenesis can promote the progression from adaptive cardiac hypertrophy to heart failure. In this regard, clinical heart failure is associated with risk factors that are also known to contribute to endothelial dysfunction and impaired angiogenic responses including diabetes, dyslipidemia, and hypertension. Thus further elucidation of the reciprocal crosstalk mechanisms between cardiomyocytes and endothelial cells will be of particular interest and may lead to novel therapeutic strategies for heart diseases.

Address correspondence to: Kenneth Walsh, Molecular Cardiology Unit, Whitaker Cardiovascular Institute, Boston University School of Medicine, 715 Albany Street, Boston, Massachusetts 02118, USA. Phone: (617) 414-2390; Fax: (617) 414-2391; E-mail: kxwalsh@bu.edu.

1. Molkentin, J.D., and Dorn, I.G., 2nd. 2001. Cytoplasmic signaling pathways that regulate cardiac hypertrophy. Annu. Rev. Physiol. 63:391-426.

2. Richey, P.A., and Brown, S.P. 1998. Pathological versus physiological left ventricular hypertrophy: a review. J. Sports Sci. 16:129-141.

3. Hudlicka, O., Brown, M., and Egginton, S. 1992. Angiogenesis in skeletal and cardiac muscle. Physiol. Rev. 72:369-417.

4. Shiojima, I., et al. 2005. Disruption of coordinated cardiac hypertrophy and angiogenesis contributes to the transition to heart failure. J. Clin. Invest. 115:2108-2118. doi:10.1172/JCI24682.

5. Izumiya, Y., et al. 2006. Vascular endothelial growth factor blockade promotes the transition from compensatory cardiac hypertrophy to failure in response to pressure overload. Hypertension. 47:887-893.

6. Friehs, I., et al. 2006. Vascular endothelial growth factor prevents apoptosis and preserves contractile function in hypertrophied infant heart. Circulation. 114:I290-I295.

7. Friehs, I., et al. 2006. Vascular endothelial growth factor delays onset of failure in pressure-overload hypertrophy through matrix metalloproteinase activation and angiogenesis. Basic Res. Cardiol. 101:204-213.

8. Sano, M., et al. 2007. p53-induced inhibition of Hif- 1 causes cardiac dysfunction during pressure overload. Nature. 446:444-448.

9. Karch, R., et al. 2005. The spatial pattern of coronary capillaries in patients with dilated, ischemic, or inflammatory cardiomyopathy. Cardiovasc. Pathol. 14:135-144.

10. Abraham, D., et al. 2000. Selective downregulation of VEGF-A(165), VEGF-R(1), and decreased capillary density in patients with dilative but not ischemic cardiomyopathy. Circ. Res. 87:644-647.

11. Shiojima, I., and Walsh, K. 2006. Regulation of cardiac growth and coronary angiogenesis by the Akt/ PKB signaling pathway. Genes Dev. 20:3347-3365.

12. Heineke, J., et al. 2007. Cardiomyocyte GATA4 
functions as a stress-responsive regulator of angiogenesis in the murine heart. J. Clin. Invest. 117:3198-3210. doi:10.1172/JCI32573.

13. Oka, T., Xu, J., and Molkentin, J.D. 2007. Reemployment of developmental transcription factors in adult heart disease. Semin. Cell Dev. Biol. 18:117-131.

14. Morisco, C., et al. 2001. Glycogen synthase kinase 3 beta regulates GATA4 in cardiac myocytes. J. Biol. Chem. 276:28586-28597.

15. Charron, F., et al. 2001. Tissue-specific GATA factors are transcriptional effectors of the small GTPase RhoA. Genes Dev. 15:2702-2719.

16. Liang, Q., et al. 2001. The transcription factor GATA4 is activated by extracellular signal-regulated kinase 1- and 2-mediated phosphorylation of serine 105 in cardiomyocytes. Mol. Cell. Biol. 21:7460-7469.

17. Brugarolas, J.B., Vazquez, F., Reddy, A., Sellers, W.R., and Kaelin, W.G., Jr. 2003. TSC2 regulates
VEGF through mTOR-dependent and -independent pathways. Cancer Cell. 4:147-158.

18. Takahashi, A., et al. 2002. Myogenic Akt signaling regulates blood vessel recruitment during myofiber growth. Mol. Cell. Biol 22:4803-4814.

19. Tirziu, D., et al. 2007. Myocardial hypertrophy in the absence of external stimuli is induced by angiogenesis in mice. J. Clin. Invest. 117:3188-3197. doi:10.1172/JCI32024.

20. Carmeliet, P., et al. 1999. Impaired myocardial angiogenesis and ischemic cardiomyopathy in mice lacking the vascular endothelial growth factor isoforms VEGF164 and VEGF188. Nat. Med. 5:495-502.

21. Giordano, F.J., et al. 2001. A cardiac myocyte vascular endothelial growth factor paracrine pathway is required to maintain cardiac function. Proc. Natl. Acad. Sci. U. S. A. 98:5780-5785.

22. Dor, Y., et al. 2002. Conditional switching of VEGF provides new insights into adult neovas- cularization and pro-angiogenic therapy. EMBOJ. 21:1939-1947.

23. Folkman, J. 1998. Is tissue mass regulated by vascular endothelial cells? Prostate as the first evidence. Endocrinology. 139:441-442.

24. Folkman, J. 2001. Angiogenesis. In Harrison's principles of internal medicine. 15th edition. E. Braunwald, et al., editors. McGraw-Hill. New York, New York, USA. 517-530.

25. Rupnick, M.A., et al. 2002. Adipose tissue mass can be regulated through the vasculature. Proc. Natl. Acad. Sci. U. S. A. 99:10730-10735.

26. Franck-Lissbrant, I., Haggstrom, S., Damber, J.E., and Bergh, A. 1998. Testosterone stimulates angiogenesis and vascular regrowth in the ventral prostate in castrated adult rats. Endocrinology. 139:451-456.

27. LeCouter, J., et al. 2003. Angiogenesis-independent endothelial protection of liver: role of VEGFR-1. Science. 299:890-893.

\title{
Kidney kinase network regulates renal ion cotransport
}

\author{
Nati Hernando, Carsten Wagner, Jürg Biber, and Heini Murer
}

Institute of Physiology, University of Zurich, Zurich, Switzerland.

\begin{abstract}
Protein kinases catalyze the phosphorylation of serine/threonine or tyrosine residues, which may directly alter a protein's functional properties. Kinases can also regulate protein functions indirectly, for example, by controlling the composition and/or subcellular localization of members of multiprotein complexes that associate with the regulated protein. In this issue of the JCI, two separate studies by Weinman et al. and Yang et al. examine the second of these two modes of kinase-mediated regulation and demonstrate the effects of kinases on two $\mathrm{Na}^{+}$-driven renal cotransporters (see the related articles beginning on pages 3403 and 3412). Their results reveal important implications for phosphate and salt homeostasis, respectively.
\end{abstract}

Homeostatic control of body fluids is achieved by, among other mechanisms, a variety of solute transporters that operate according to the body's needs. Thus, the function of these transporters is modulated by diverse regulatory systems. Frequently, the final event in a hormonally stimulated signaling cascade involves activation of protein kinases that modify transporter activities by changing the characteristics or the subcellular localization of the transporters. Such alterations

Nonstandard abbreviations used: BBM, brush border membrane; DCT, distal convoluted tubule; NCC, thiazide-sensitive $\mathrm{Na}-\mathrm{Cl}$ cotransporter; NHERF-1, sodiumhydrogen exchanger regulatory factor- $1 ; \mathrm{Npt} 2 \mathrm{a}, \mathrm{Na}^{+}-\mathrm{P}_{\mathrm{i}}$

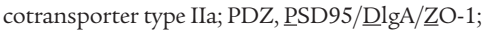
$\mathrm{P}_{\mathrm{i}}$, inorganic phosphate; $\mathrm{PT}$, proximal tubule; $\mathrm{PTH}$, parathyroid hormone; WNK, with no lysine ( $\underline{\mathrm{K}})$.

Conflict of interest: The authors have declared that no conflict of interest exists.

Citation for this article: J. Clin. Invest. 117:3179-3182 (2007). doi:10.1172/JCI33859. can be achieved by direct phosphorylation of the transporter itself or via phosphorylation of proteins that are part of heteromultimeric complexes.

Protein kinase-mediated activation or inhibition of many hormonally controlled transporters have been described, yet the final targets in these signaling pathways and/or the underlying regulatory mechanisms have not yet been identified. The work presented in this issue of the JCI in the articles by Weinman et al. (1) and Yang et al. (2) now sheds some mechanistic light on how the activity and/or abundance of ion transporters are hormonally controlled by protein complexes that contain protein kinases. These two reports respectively address the regulation of two ion transporters that are predominantly expressed in kidney: the parathyroid hormone-regulated (PTH-regulated) $\mathrm{Na}^{+}-\mathrm{P}_{\mathrm{i}}$ cotransporter type IIa (Npt2a; encoded by SLC34A1 and also known as NaPi-IIa) (3) and the thiazide-sensitive $\mathrm{Na}-\mathrm{Cl}$ cotransporter (NCC; encoded by SLC12A3 and also known as TSC) (4). The NCC is located on the luminal side of distal convoluted tubule (DCT) cells, whereas Npt2a associates with the brush border membranes (BBMs) of proximal tubules (PTs) (3, 5). Apical expression of NCC and Npt2a is under the control of kinases such as WNK kinases (with no lysine $[\underline{K}]$ in their catalytic domain II) and PKA/PKC, respectively. Interestingly, neither WNK nor PKA/PKC have been reported to phosphorylate these transporters directly $(3,6-8)$.

\section{Npt2a and NCC: function and dysfunction}

Npt2a mediates $\mathrm{Na}^{+}$-coupled, electrogenic reabsorption of inorganic phosphate $\left(\mathrm{P}_{\mathrm{i}}\right)$. The abundance of the Npt2a protein is regulated by dietary phosphate intake, by phosphaturic PTH, and by different phosphatonins $(3,8,9)$. The importance of Npt2a in phosphate homeostasis was documented in an $\mathrm{Npt2}^{-/-}$mouse model that is characterized by hypophosphatemia (abnormally decreased level of phosphate in the blood) and hyperphosphaturia (increased excretion of phosphate in the urine) due to reduced $\mathrm{P}_{\mathrm{i}}$ reabsorption (10). Npt2a expression is reduced in animal models of X-linked hypophosphatemia, and a similar defect may also be implicated 